\title{
Tidally-forced flow in a rotating, stratified, shoaling basin
}

\author{
Kraig B. Winters \\ Scripps Institution of Oceanography \\ University of California San Diego
}

\begin{abstract}
Baroclinic flow of a rotating, stratified fluid in a parabolic basin is computed in response to barotropic tidal forcing using the nonlinear, non-hydrostatic, Boussinesq equations of motion. The tidal forcing is derived from an imposed, boundary-enhanced free-surface deflection that advances cyclonically around a central amphidrome. The tidal forcing perturbs a shallow pycnocline, sloshing it up and down over the shoaling bottom. Nonlinearities in the near-shore internal tide produce an azimuthally independent 'set-up' of the isopycnals that in turn drives an approximately geostrophically balanced, cyclonic, near-shore, sub-surface jet. The sub-surface cyclonic jet is an example of a slowly evolving, nearly balanced flow that is excited and maintained solely by forcing in the fast, super-inertial frequency band. Baroclinic instability of the nearly balanced jet and subsequent interactions between eddies produce a weak transfer of energy back into the inertia-gravity band as swirling motions with super-inertial vorticity stir the stratified fluid and spontaneously emit waves. The sub-surface cyclonic jet is similar in many ways to the poleward flows observed along eastern ocean boundaries, particularly the California Undercurrent. It is conjectured that such currents may be driven by the surface tide rather than by winds and/or along-shore pressure gradients.
\end{abstract}

Keywords: internal tide, cyclonic jet, baroclinic instability, poleward eastern boundary currents

\section{Introduction}

Oceanic internal tides are inertia-gravity waves at tidal frequencies, usually the semi-diurnal $M_{2}$, excited by the barotropic tide as it induces vertical

Email address: kraig@coast.ucsd.edu () 
displacement of stably-stratified fluid in the vicinity of topographic variability. Because $M_{2}$ lies within the inertia-gravity wave band $f$ to $N$ defined by the linear dispersion relation, the interaction between barotropic tidal flows and topography is able to generate freely-propagating internal tides that radiate away from generation sites. Here $f$ and $N$ are the local inertial and buoyancy frequencies respectively. Previous studies have focused on internal tides generated at isolated topographic features within basins such as ridge systems like the Hawaiian Ridge (Merrifield et al., 2001; Merrifield and Holloway, 2002; Rudnick et al., 2003) and the Luzon Strait (Mercier et al., 2013; Li and Farmer, 2011; Zhao, 2014) or at continental slope-shelf breaks where the topographic slope transitions from super- to sub-critical values with respect to the inclination angle of energy propagation for internal tides (Huthnance, 1989; Pingree and New, 1992; Gerkema et al., 2004). Near ridges the interest is mostly in energetic, low-vertical-mode internal tides that evolve as they propagate away to the far field, e.g. Hazewinkel and Winters (2011), while at shelf breaks studies tend to focus on the smaller scale components that form tidal beams.

A central issue is the energy balance in the conversion of barotropic to baroclinic motions with particular emphasis on the relative magnitudes of the radiating and trapped baroclinic motions. A better understanding of the environmental parameters and physical mechanisms that produce trapped and radiating motions is necessary in order to better understand and predict the intensity and spatial distribution of tidally driven mixing and dissipation in the ocean, e.g. Garrett (2003).

In this study, we consider the effect of an idealized free-surface tide in the absence of isolated topographic features and ask, what is the nature of the baroclinic response in a shoaling, rotating, stratified basin? In this context, the shoaling, near-shore region is the generation site for inertia-gravity waves and the overall baroclinic response consists of both waves that radiate toward the basin interior and trapped, near-shore motions at frequencies less than $f$. We compute the response of a continuously stratified fluid in a rotating, parabolic basin to idealized tidal forcing. The tide is modeled as an imposed, boundary-enhanced free-surface deflection that rotates cyclonically at frequency $\sigma>f$ about a single amphidrome located at the basin center. The ambient stratification consists of a thin mixed layer and a sharp, near-surface, pycnocline with $N>>\sigma$ overlying weak stratification with $N$ slightly less than $\sigma$ at depth. The forcing excites boundary intensified motions that advance cyclonically around the basin, sloshing the pycnocline up and down over the shoaling bottom. The azimuthal wavelength of these motions matches the circumference of the basin. 
The fast time scales of the problem are associated with the tidal and inertial frequencies $\sigma$ and $f$. We will see that a slow time scale emerges that is associated with the interaction and pairing of the near-shore eddies. The fast and slow time scales are dynamically linked in this flow by the eddies themselves which have a vertical vorticity magnitude comparable to $f$.

Our approach here is to convert the idealized tidal model into a time and space dependent horizontal body force that is then applied to a rigid-lid numerical model of rotating, stratified flow in a parabolic basin. In taking this approach, we relax the various assumptions that might make analytical calculations tractable, namely the assumptions of linearity, hydrostaticity, uniform basin depth, and simple two-layer stratification, and compute the baroclinic response to the idealized tidal forcing via non-hydrostatic numerical simulation.

The remainder of the paper is organized as follows. The idealized tidal model is described in section 2 followed by a description of the mathematical model and a brief sketch of its numerical solution in section 3. The dimensionless parameters and the asymptotic regimes of the flow are given in section 4 . Simulation results are presented in 5 followed by a discussion in 6 .

\section{Experimental setup}

We consider a parabolic basin with a stress-free rigid lid in a rotating reference frame as shown in figure 1. A simple model of a free surface tide is specified by

$$
\eta=\eta_{o}(r) \cos (\theta-\sigma t)
$$

where $\eta$ is the deviation of the surface elevation from its equilibrium value, $r$ is the radial distance from the domain center $\left(x_{o}, y_{o}\right), \theta$ is the polar angle that increases in the counter-clockwise or cyclonic direction, and $\sigma$ is the forced tidal frequency. The shape function $\eta_{o}(r)$ is taken as a polynomial with $\gamma=3$,

$$
\eta_{o}(r)=\eta_{\max }\left(\frac{r}{R}\right)^{\gamma} .
$$

The free surface deflection $\eta$ is maximal at the boundary, decays to zero in the interior and has a phase that propagates in the counter-clockwise direction with a period of $2 \pi / \sigma$ (see figure 2 ). From this simple model, we define a body force

$$
\mathbf{F}(x, y, z, t)=-g \nabla \tilde{\eta} .
$$

Here $\mathbf{F}$ is written as an explicit function of $z$ and $\eta$ with a tilde to indicate that, near the curved boundary, the component of $\nabla \eta$ normal to the 

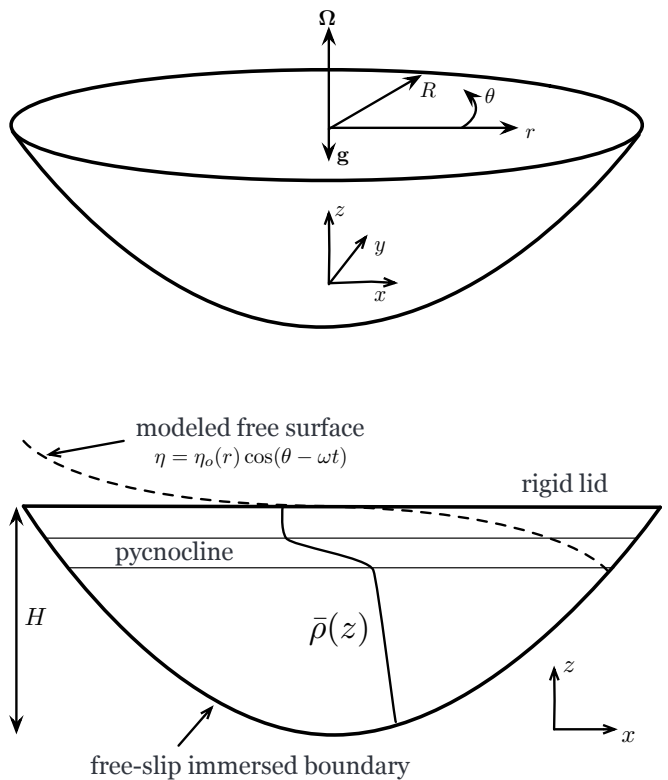

Figure 1: Schematic of a parabolic basin with surface radius $R$ and depth $H$. An initially quiescent fluid is set into motion by a body force $\mathbf{F}$, which models forcing by a barotropic tide advancing cyclonically along the coastline with an amphidrome at the basin center. The stratification is characterized by a surface mixed layer, a strong pycnocline and a weak stratification at depth.

immersed boundary is smoothly tapered to zero over a scale that is comparable to the grid spacing. We regard the resulting $\mathbf{F}$ as an externally imposed body force that models the influence of a barotropic tide for an irregularly shaped basin with a rigid lid. Our objective is to determine the internal response to this forcing.

\section{Mathematical model}

\subsection{Basin shape}

The basin shape is defined within a cubic computational domain of dimensions $L_{x}, L_{y}$ and $L_{z}$ by the height of the bottom boundary $h(x, y)$ where

$$
h(x, y)=h_{o}+\frac{r^{2}}{\alpha^{2}}
$$

Here $\alpha^{2}$ is equal to $\frac{1}{4}\left(0.95 L_{x}\right)^{2} / L_{z}$. For small but finite $h_{o}>0$, the deepest point in the basin is slightly above $z=0$ and the basin outcrops the upper 
surface $z=L_{z}$ slightly inside the vertical computational boundaries. The effective dimensions of the basin are thus $R$ and $H$, as shown in figure 1, which are slightly smaller than $L_{x} / 2=L_{y} / 2$ and $L_{z}$ respectively. In presenting results, we will shift the coordinate origin to the point $\left(x_{o}, y_{o}, z-\right.$ $h_{o}$ ) and normalize horizontal and vertical positions by $R$ and $H$ respectively.

\subsection{Equations of motion}

The flow is modeled using the Boussinesq equations of motion for a rotating stratified fluid on an $f$-plane augmented with the tidal body force F

$$
\frac{\partial}{\partial t} u+\vec{u} \cdot \nabla u-f v=-\frac{1}{\rho_{o}} \frac{\partial p^{\prime}}{\partial x}+\mathbf{F} \cdot \hat{x}+\mathcal{D}_{u}
$$

$$
\frac{\partial}{\partial t} v+\vec{u} \cdot \nabla v+f u=-\frac{1}{\rho_{o}} \frac{\partial p^{\prime}}{\partial y}+\mathbf{F} \cdot \hat{y}+\mathcal{D}_{v}
$$

$$
\frac{\partial}{\partial t} w+\vec{u} \cdot \nabla w+\frac{g}{\rho_{o}} \rho^{\prime}=-\frac{1}{\rho_{o}} \frac{\partial p^{\prime}}{\partial z}+\mathcal{D}_{w}
$$

$$
\frac{\partial}{\partial t} \rho+\vec{u} \cdot \nabla \rho=\mathcal{D}_{\rho}
$$

$$
\nabla \cdot \vec{u}=0 .
$$

Here $\hat{x}$ and $\hat{y}$ are horizontally oriented unit vectors, $f$ is the Coriolis parameter and $g$ is the gravitational acceleration. The $\mathcal{D}$ terms are dissipation operators discussed further below.

The perturbation density $\rho^{\prime}$ is defined with respect to a time independent ambient vertical profile $\bar{\rho}$ where

$$
\rho=\bar{\rho}(z)+\rho^{\prime}(x, y, z, t)
$$

and $\bar{\rho}(z)$ is the initial density profile. Pressure is decomposed into a depth dependent part that is in hydrostatic balance with $\bar{\rho}$ and a perturbation component $p^{\prime}$ defined via

$$
p=-g \int^{z} \bar{\rho}(\tilde{z}) \mathrm{d} \tilde{z}+p^{\prime}(x, y, z, t) .
$$

The hydrostatic balance drops out of Eq. (7) in the usual way and so only $\rho^{\prime}$ appears in that equation while $p^{\prime}$ appears throughout. 
The $\mathcal{D}$ terms are a combination of Laplacian operators with molecular viscosity and "hyper-diffusivity" operators. For example

$$
\mathcal{D}_{u}=\nu \nabla^{2} u+\nu_{h}\left(\frac{\partial^{6}}{\partial x^{6}}+\frac{\partial^{6}}{\partial y^{6}}\right) u+\nu_{v}\left(\frac{\partial^{6}}{\partial z^{6}}\right) u .
$$

The operators for the other fields are similar. The coefficients of the "hyperdiffusivity" operators have dimensions $m^{6} / s$ and are specified so that the relatively weak downscale variance cascades are efficiently removed at the smallest resolvable scales while permitting very small molecular diffusivities to be prescribed. This is important here as we wish to conduct numerical experiments over hundreds of forcing periods without having the ambient stratification diffuse so much that the wave environment changes considerably. Specifying only Laplacian diffusion is problematic: diffusivity values large enough to ensure numerical stability diffuse the ambient stratification excessively while diffusivities small enough to nearly maintain the initial stratification are not sufficient to arrest the downscale cascade and maintain numerical stability. Because the underlying numerical method is based on Fourier expansions, these dissipation operators are easily and accurately computed.

\subsection{Boundary conditions}

The upper boundary is a rigid lid with $w, \frac{\partial u}{\partial z}, \frac{\partial v}{\partial z}$ and $\frac{\partial \rho}{\partial z}$ all prescribed to be zero. To avoid having to resolve small viscous boundary layers, the curved bottom boundary is taken as a free-slip wall with $\vec{u} \cdot \hat{n}=0$, where $\hat{n}$ is the local unit vector normal to the boundary. No boundary condition is imposed on $\rho$ at this boundary. These are the exact boundary conditions desired in the limiting case of an inviscid, nondiffusive fluid. We recall for example that, for an ideal fluid, the exact solution for a linear internal Kelvin wave at a vertical sidewall (Gill, 1982; Csanady, 1967) has nonvanishing normal gradients of both the along-wall speed and density. In deriving these solutions, the only boundary condition at the wall is the no penetration condition. Stress and scalar diffusion through the wall vanish by virtue of vanishing molecular diffusivities, not vanishing normal gradients of the solutions. We adopt this approach here under the idealization that unless a no-slip condition is imposed and the tangential flow is required to vanish at the curved boundary, the action of the viscous terms is negligible and thus vanishing normal flow at the wall is the only allowable boundary condition to prescribe. This approach then permits small but physically negligible stresses and scalar fluxes at the boundary when the molecular diffusivities are taken sufficiently small as discussed in section 4 . 


\subsection{Initial conditions}

The experiments start from a state of rest at $t=0$ :

$$
\vec{u}(\vec{x}, 0)=0 .
$$

The initial density profile consists of a surface mixed layer, a thin, strongly stratified pycnocline and a weaker stratification at depth. To specify the initial profile, we first define several parameters. Let $z_{i}$ and $\delta$ be the midheight and thickness of the pycnocline respectively and $z_{1}=z_{i}+\delta / 2$ and $z_{2}=z_{i}-\delta / 2$. Let $\rho_{\max }, \rho_{1}$ and $\rho_{2}$ be the density values at $z=0$, at $z_{1}$ and at $z_{2}$ respectively. We then define the characteristic magnitude of the density gradients in the deep basin and in the pycnocline as

$$
c_{1}=\frac{\rho_{\max }-\rho_{2}}{z_{1}} \text { and } c_{2}=\frac{\rho_{2}-\rho_{1}}{\delta}
$$

as shown in figure 2 . Introducing a small transition scale $\beta=\delta / 10$, and a superposition of approximate step functions using hyperbolic tangents, we integrate in $z$ to obtain

$$
\begin{aligned}
\bar{\rho}(z)= & \frac{c_{1}}{2}\left(\beta \log \left(\cosh \left(\left(z-z_{1}\right) / \beta\right)\right)-z\right) \\
& +\frac{c_{2}}{2}\left(\beta \log \left(\cosh \left(\left(z-z_{1}\right) / \beta\right)\right)\right) \\
& -\frac{c_{2}}{2}\left(\beta \log \left(\cosh \left(\left(z-z_{2}\right) / \beta\right)\right)\right)-\bar{\rho}_{s}
\end{aligned}
$$

where $\bar{\rho}_{s}$ is a constant chosen such that the value of $\bar{\rho}$ at the surface is equal to zero. We set $\rho(\vec{x}, 0)=\bar{\rho}(z)$, which gives the initial profiles shown in figure 2. The characteristic squared buoyancy frequencies in the pycnocline and in the deep basin are given by

$$
N_{\text {deep }}^{2}=\frac{g}{\rho_{o}} c_{1} \text { and } N_{\text {pyc }}^{2}=\frac{g}{\rho_{o}} c_{2} .
$$

As indicated in figure 2, the forcing frequency $\sigma$ is less than $N$ within the main pycnocline and greater than $N$ below.

\subsection{Numerical solution}

The equations of motion are solved using trigonometric expansions for the dependent variables in a cubic domain of size $L_{x}, L_{y}$ and $L_{z}$. All variables are expanded in cosine series in both horizontal directions. In the vertical, $u$, $v$ and $\rho$ are expanded in cosine series while $w$ is expanded in a sine series and so the prescribed boundary conditions at the rigid lid are satisfied implicitly. 

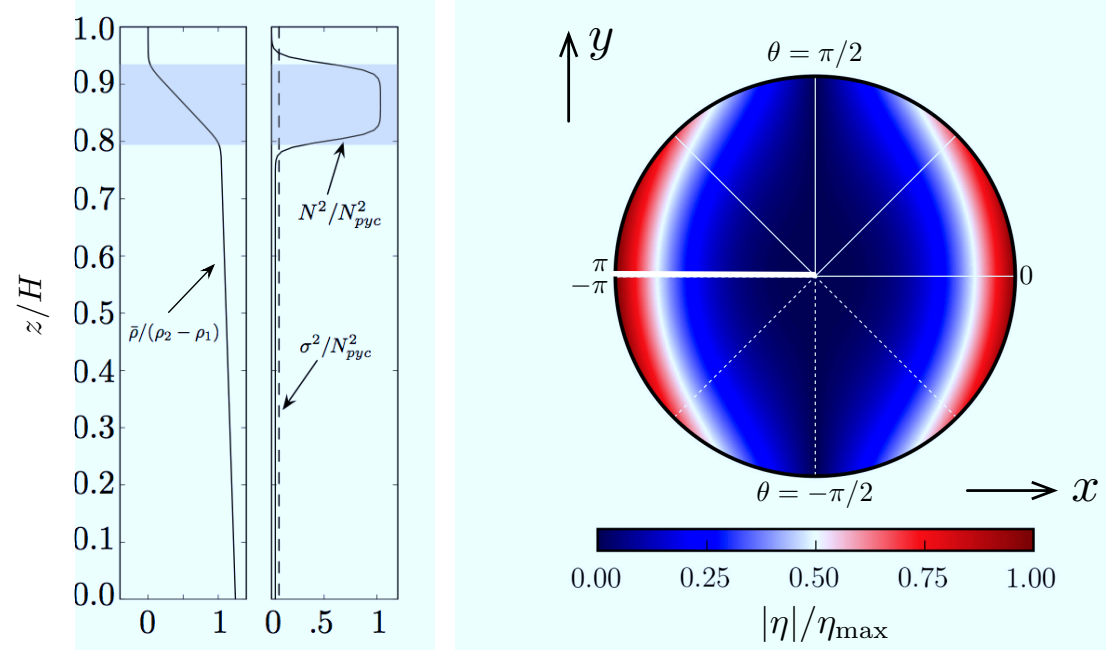

Figure 2: Left: Initial density profile $\bar{\rho}(z)$ and corresponding profile of the squared buoyancy frequency $N^{2}$. The depth range $z_{2}<z<z_{1}$ is shaded. The dashed vertical line shows the square of the tidal frequency $\sigma$. Right: Magnitude of $\eta$ (color) at $t=0$, the convention for the azimuthal angle $\theta$ and the cyclonically rotating tidal phase lines. 
The boundary conditions on the curved bottom boundary are implemented using an immersed boundary approach (Winters and de la Fuente, 2012).

The equations are integrated for $O(250)$ forcing periods using a $2-\mathrm{D}$ domain decomposition on distributed memory machines with 256 or 512 processors. The primary output consists of data, $u, v, w, \rho^{\prime}$ and $p^{\prime}$, from horizontal planes just below the base of the mixed layer, within, and below the pycnocline, and on the two vertical planes $y=0$ and $x=0$. Data from these two-dimensional planes are saved $\approx 100$ times per forcing period. Full three-dimensional snapshots are saved less frequently and can be used to restart the simulations to generate different or additional data as desired.

\section{Ordering of scales}

The dimensionless parameters defining the simulation are listed in Table 1. Here we summarize briefly the asymptotic sizes of a few of the key dimensionless parameters. First, $N_{\text {pyc }} / f=O(10)$ and $\sigma / f>2$. Thus, forcing at frequency $\sigma$ can potentially excite free internal waves at both higher and lower frequencies and the overall frequency bandwidth is comparable to, but smaller than, that in the mid-latitude ocean thermocline where $N / f \approx 50$.

The external Rossby radius of deformation $a_{\text {ext }}$ is imposed implicitly via $\eta_{o}(r)$ and is smaller than, but of the same order, as the surface basin radius $R$. The internal Rossby radius $a_{\mathrm{int}} \approx c / f \approx 0.1 R$, i.e. is asymptotically small compared to $R$ but larger than typical ocean values. Here $c=\sqrt{\frac{g^{\prime} h_{1} h_{2}}{H}}$, $h_{1}$ is the depth of the pycnocline center, $h_{2}=H-h_{1}$ and $g^{\prime}=g \Delta \rho / \rho_{o}$ with $\Delta \rho=\rho_{2}-\rho_{1}$, the density difference across the pycnocline. Increasing both $N_{\text {pyc }} / f$ and $R$ would yield a wider band of internal wave frequencies and a larger scale separation between the internal and external deformation radii, but at considerably greater computational expense.

The strength of the tidal forcing is quantified by comparing the characteristic magnitude of the imposed vertical acceleration of the pycnocline to the restoring force per unit mass induced when this occurs, $g^{\prime}$. The characteristic horizontal acceleration is $|g \nabla \eta| \approx g \eta_{\max } / R$. Horizontally accelerated flow is deflected vertically owing to the parabolic basin shape and so the characteristic vertical acceleration induced by the tidal forcing is given by $g\left(\eta_{\max } / R\right)(H / R)$, where $H / R$ is the overall basin slope. The ratio of the forced vertical acceleration to the restoring force per unit mass $g^{\prime}$ is then $\left(\rho_{o} / \Delta \rho\right)\left(\eta_{\max } / R\right)(H / R)$, a measure of how strongly the pycnocline is being vertically displaced by the forcing. Here we take

$$
\frac{\rho_{o}}{\Delta \rho} \frac{\eta_{\max }}{R} \frac{H}{R}<<1
$$


Table 1: Dimensionless parameters defining the flow regime and the model resolution. Symbols are defined in section 4.

\begin{tabular}{|c|c|l|}
\hline parameter & value & comment \\
\hline$N_{\text {pyc }} / f$ & 11.6 & strongly stratified pycnocline \\
\hline$N_{\text {deep }} / N_{\text {pyc }}$ & 0.19 & weak stratification at depth \\
\hline$\sigma / f$ & 2.7 & $2 f<N_{\text {deep }}<\sigma<N_{p y c}$ \\
\hline$\delta / H$ & 0.13 & thin pycnocline \\
\hline$h_{1} / h_{2}$ & 0.06 & near-surface pycnocline \\
\hline$H / R$ & 0.12 & shallow basin \\
\hline$\left(\rho_{o} / \Delta \rho\right)\left(\eta_{\max } / R\right)(H / R)$ & 0.046 & weak tidal forcing \\
\hline$[\eta / \partial \eta / \partial r] / R$ & $\approx 1$ & external Rossby deformation radius \\
\hline$a_{\text {int }} / R=(c / f) / R$ & 0.12 & internal Rossby deformation radius \\
\hline$\delta^{2} f / \nu$ & $4.6 \times 10^{3}$ & Laplacian dissipation is negligible \\
\hline$\left(\nu_{h} k_{*}^{6} \Delta t\right)^{-1}$ & 5 & $k_{*}$ is horizontal Nyquist wavenumber \\
\hline$\left(\nu_{v} m_{*}^{6} \Delta t\right)^{-1}$ & 5 & $m_{*}$ is vertical Nyquist wavenumber \\
\hline$\Delta x / R=\Delta y / R$ & 0.004 & horizontal grid spacing \\
\hline$\Delta z / \Delta x$ & 0.22 & grid aspect ratio \\
\hline$\sigma \Delta t$ & $0.025,0.0125$ & time step \\
\hline
\end{tabular}

and in this sense, internal waves in the basin are weakly forced by the tide.

The importance of dissipation is quantified by two dimensionless parameters reflective of the two operators in Eq. (12). $\delta^{2} f / \nu$ is the ratio of the time required to diffuse momentum or mass across the pycnocline thickness $\delta$ to the inertial time scale $1 / f$. This quantity is $O\left(10^{3}\right)$ and so the Laplacian terms are negligible over the simulation time of the experiments. $1 /\left(\nu_{h} k_{*}^{6} \Delta t\right)$ is the decay time associated with the sixth order operator at the spatial scale corresponding to the maximum resolved horizontal wavenumber $k_{*}$ divided by the time step $\Delta t$. Similar quantities define the effective strength of the vertical diffusion of momentum and the horizontal and vertical diffusion of mass. These quantities prescribed to be $O(1)$ so that variance that is transferred from larger scales to the smallest resolvable scales is dissipated quickly, i.e. within a few time steps. The influence of these higher order operators, however, is strongly restricted to motions near the grid scale. Motions at scales of $O(10 \Delta x)$ for example are dissipated on a time scale $10^{6}$ times longer. Thus, except for a weak filtering effect near the grid scale, the computed flow is governed by equations that are essentially inviscid and non-diffusive. 


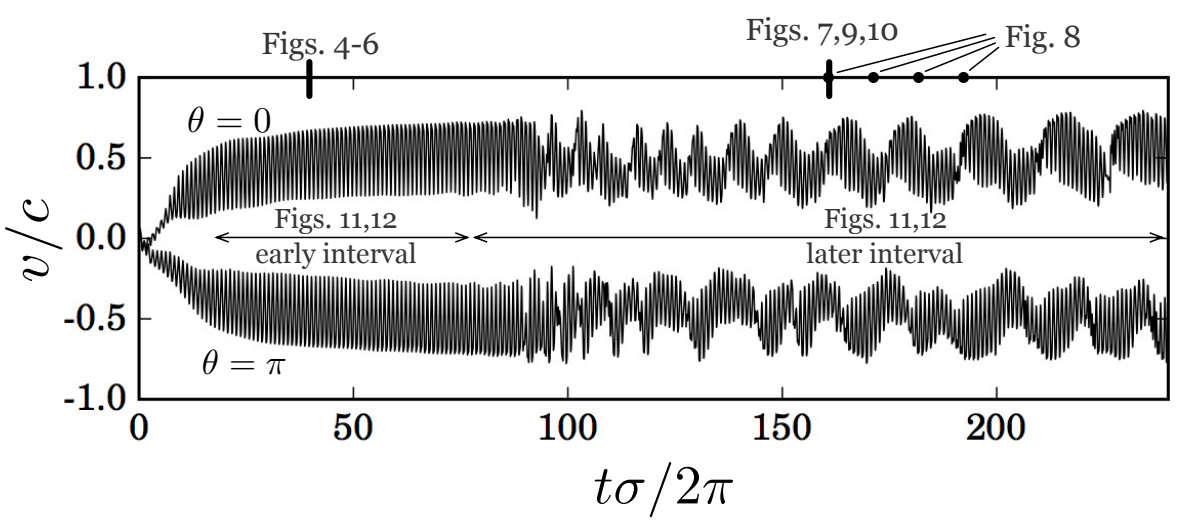

Figure 3: Time series of along shore speed $v$ at $y=0, x / R= \pm 0.93$ and $z / H=0.925$. At $\theta=\pi, v$ is the negative of the azimuthal speed $v_{\theta}$, which is positive for cyclonic flow.

\section{Results}

Results from a single experiment with parameters listed in Table 1 are now presented in some detail. We defer a systematic parameter study to future work and focus on presenting and explaining the relatively rich flow field that develops.

\subsection{Overview: near-shore time series}

Figure 3 shows near-shore time series of the $y$ component of velocity $v$ taken from within the pycnocline at $\theta=0$ and $\pi$. The time series are normalized by the wave speed $c$ used to define the internal Rossby deformation radius $a_{\text {int }}$ in section 4 . Both time series show high frequency fluctuations on the 'fast' time scale of the tidal forcing. The magnitude of these fluctuations increases over about the first 20 tidal periods and then remains approximately constant for about 70 periods. Very quickly after startup however, the flow develops a residual component, becoming always positive at $\theta=0$ and always negative at $\theta=\pi$. The time averaged flow at both locations is in the counterclockwise or cyclonic sense. After about the first 20 tidal periods, the along-shore currents at these locations oscillate regularly about a slowly increasing mean speed. After about 90 tidal periods however, both time series become much more irregular, though the time average currents change very little.

Except for temporal phase, the flow prior to $t \sigma / 2 \pi \approx 90$ is essentially independent of $\theta$ once it is resolved into azimuthal and radial components. 


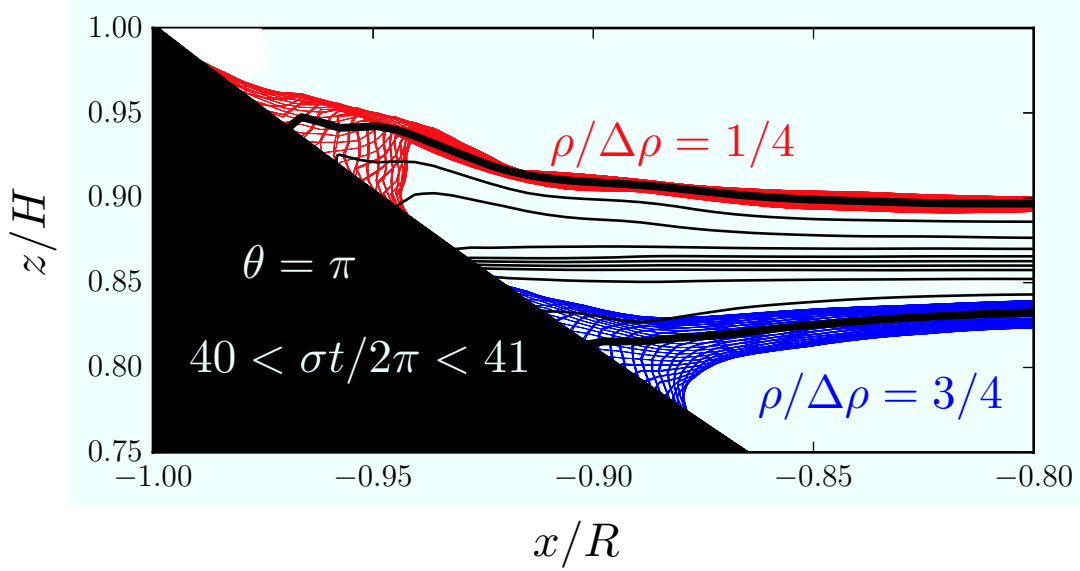

Figure 4: Black: Mean isopycnal positions at $\theta=\pi$, averaged over the tidal period $40<t \sigma / 2 \pi<41$. Colored isopycnals show the instantaneous positions corresponding to those shown with heavy black lines throughout the period. Here $\Delta \rho$ is the initial density jump across the pycnocline and contour values are uniformly spaced within the range $1 / 4 \leq \rho / \Delta \rho \leq 3 / 4$.

In displaying the cross-shore structure of the flow, we show results taken from the region surrounding the location of the $\theta=\pi$ time series shown in figure 3. For cross reference with these time series, the time values for the remaining figures are marked in the figure.

\subsection{Internal wave 'set-up'}

The cross-shore structure of the near-shore isopycnals is shown in figure 4. The black contours are isopycnals computed after time averaging the density field over the $41^{\text {st }}$ tidal forcing period indicated in figure 3. During this time interval the tidal fluctuations are regular and the magnitude of the near- and along-shore flow $v$ is accelerating only slowly. The density values are normalized by the initial density contrast across the pycnocline $\Delta \rho$ and the time averaged positions of $\rho / \Delta \rho=1 / 4$ and $3 / 4$ are shown with heavy lines. Also shown are instantaneous positions of these same isopycnals at equally spaced intervals throughout the tidal cycle in red and blue respectively.

The time averaged isopycnal response is asymmetric about the midpycnocline value $\rho / \Delta \rho=1 / 2$. Near the sloping boundary the upper portion of the pycnocline is lifted significantly upward on average, while the lower portion is deflected only mildly downward. At $x / R=-.95$ for example, the time averaged upward deflection of the $\rho / \Delta \rho=1 / 4$ is approximately one 
quarter of the local depth. The upper portion of the pycnocline sloshes up and down over the sloping boundary at the tidal frequency but with residual positions that are lifted upward compared to the basin interior by an amount that is significant compared to the local depth.

The internal wave 'set-up' and 'set-down' shown in figure 4 are a consequence of the re-adjustment of mass and momentum required to cancel the Stokes drift for waves at a sloping boundary in the absence of mixing (Wunsch, 1971; Longuet-Higgins and Stewart, 1964). Based on an analytical calculation for weakly nonlinear, two-dimensional internal waves in a shoaling region with constant slope, Wunsch (1971) gives a scaling argument for the vertical setup of the isopycnals $Z_{\text {setup }}$ that can be written in terms of the magnitude $\mathcal{Z}$ of the tidal frequency isopycnal excursions and the local depth $D$

$$
Z_{\text {setup }} \approx \frac{\mathcal{Z}^{2}}{D} \quad[m] .
$$

Wunsch (1971) further argues that this can be a measurable effect in the ocean for small amplitude waves for which his theoretical calculations are formally valid and a potentially very large effect for finite amplitude internal waves.

This quantity can be estimated at $x / R=-0.95$. Choosing $\mathcal{Z} \approx D / 2$ to be the approximate vertical excursion scale of the $\rho / \Delta \rho=1 / 4$ isopycnal implies that

$$
Z_{\text {setup }} / D \approx 1 / 4,
$$

which matches the value inferred from figure 4 .

\subsection{Time averaged near-shore cyclonic jet}

The consequences of the internal wave 'set-up' induced by tidal forcing in a shoaling basin are significant owing to the effects of rotation. The time averaged upward deflection of the near-shore isopycnals shown in figure 4 occurs at all azimuthal positions. This induces an azimuthally-independent, residual cross-shore pressure gradient that drives a corresponding alongshore flow in the cyclonic direction. The residual of this cyclonic response gives rise to the offsets of the time series seen in figure 3 .

The stability of jets over sloping boundaries has been previously investigated for hydrostatic, two-layer flow (Poulin and Flierl, 2005; Poulin et al., 2014). Wind-driven, rectified flow in a rotating lake has also been studied in a two-layer framework ( $\mathrm{Ou}$ and Bennett, 1979). The flow here, however, is continually energized by the tidal forcing and is continuously stratified. Continuous stratification is likely to be important as the setup shown in 


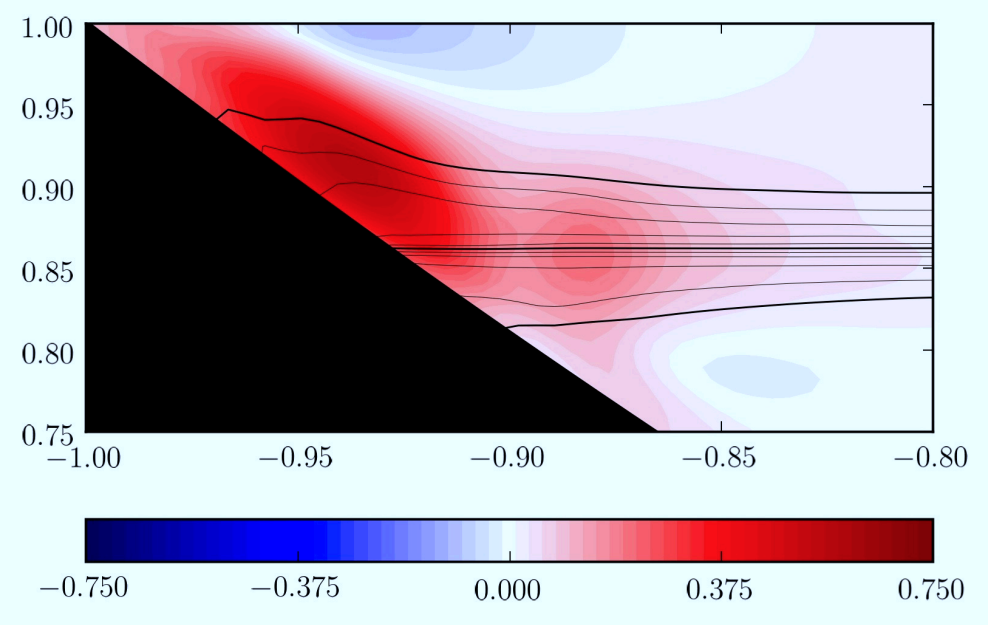

Figure 5: Azimuthal speed $v_{\theta}$ at $\theta=\pi$, averaged over the same tidal period shown in figure 4. Also shown are the time averaged isopycnals.

figures 4 and 5 has a distinct varicose character that is not captured in a two-layer formulation. In addition, in the depth range of the main pycnocline, the boundary slope $\frac{\partial h}{\partial r}$ is within about $5 \%$ of the critical angle for internal waves in a fluid characterized by $f$ and $N_{p y c}$. These waves have aspect ratios of horizontal to vertical scales of $O(1)$ and so the nearly resonant waves excited close to the boundary in this flow are non-hydrostatic.

The along-shore flow at $\theta=\pi$, averaged over the same tidal period as in figure 4 , is shown in figure 5 along with the corresponding isopycnals. The along-shore flow takes the form of a near-shore, bottom trapped cyclonic jet. The jet has a maximum speed at about $x / R=-0.95$ and weakens rapidly toward the basin interior. The horizontal scale of the jet is about $0.1 R$ which is approximately equal to the internal deformation radius $a_{\text {int }}$.

If the near-shore cyclonic jet were to be in geostrophic balance with the sloping isopycnals, then, assuming hydrostatic pressure

$$
\frac{\partial v}{\partial z} \approx-\frac{g}{\rho_{o} f} \frac{\partial \rho}{\partial x} .
$$

The degree to which this thermal wind balance holds can be estimated by 
computing the ratio of the characteristic magnitudes of each term. Taking

$$
\left|\frac{\partial v}{\partial z}\right| \approx \frac{c}{0.1 H} \quad \text { and } \quad\left|\frac{\partial \rho}{\partial x}\right| \approx \frac{.25 \Delta \rho}{0.05 R},
$$

the ratio of the size of the pressure gradient term to the vertical shear is about 1.1, suggesting the interpretation of this jet as a rotationally induced response to the tidally forced set-up of the near-shore pycnocline.

The simple view that emerges is that wave stresses induce a pycnocline setup near the sloping boundary and that this setup then produces an alongshore geostrophic jet. In detail however, a three term balance between the setup, rotation and wave stresses would be expected. Wunsch (1971) derives such a balance but notes that it is degenerate in the sense that arbitrary along-shore currents can be added that induce correspondingly arbitrary changes to the isopycnal setup.

At this point, we can now see that the $\theta=\pi$ time series in figure 3 is taken from within the near-shore cyclonic jet shown in figure 5 . The speed of the jet increases steadily over the first 20 tidal periods as the isopycnals 'set up' in response to the forcing. After the initial spinup, the speed of the jet increases only slowly, with relatively little change over the next 70 periods, consistent with the approximately geostrophic balance estimated for $40<t \sigma / 2 \pi<41$.

\subsection{Cross-shore flow}

While figure 5 shows the position and structure of the cyclonic jet when averaged over a tidal period, directly forced cross-shore motions cause the jet to move up and down on the slope at the tidal frequency. Figure 6 shows vertical profiles of the cross-shore speed $v_{r}$ at $\theta=\pi, x / R=-0.93$. The radial flow sloshes on- and off-shore with both a barotropic component of approximate magnitude $c / 4$ as well as a baroclinic, bottom intensified component. This cross-shore flow is directly forced by the tide and thus has a frequency equal to $\sigma$. Averaging over a forcing period essentially removes the cross-shore flow.

The bottom intensification of the flow is reminiscent of the vertical structure of topographic Rossby waves over a sloping bottom see e.g. (Rhines, 1970; Pedlosky, 1987). Topographic Rossby waves are, however, freely propagating modes with frequencies much smaller than $f$. For frequencies approaching $f$ from below, freely propagating bottom-trapped waves (Rhines, 1970) also decay away from the boundary, but in this limit the vertical decay scale matches the cross-shore wavelength, whereas it is much smaller than 


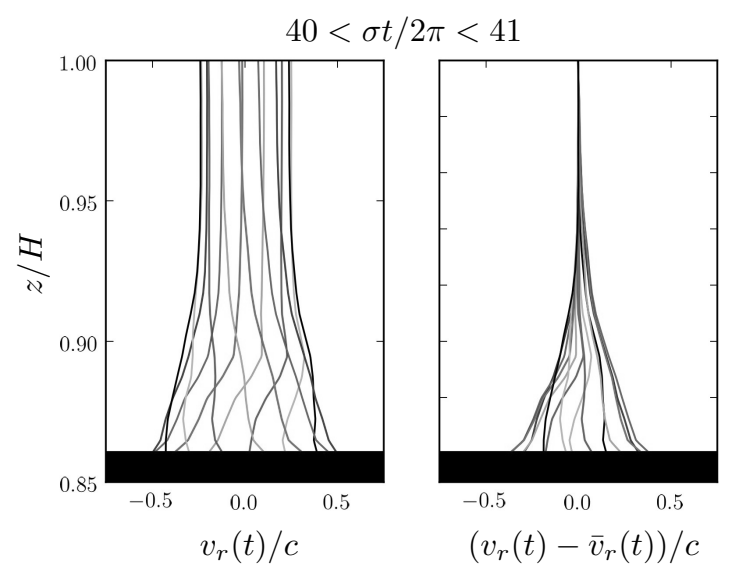

Figure 6: Profiles of cross-shore flow speed $v_{r}$ at $\theta=\pi$ and $x / R=-0.93$ for the time period $40<t \sigma / 2 \pi<41$. Left: Instantaneous profiles sampled at equal time intervals. Right: The same profiles with the instantaneous vertical mean speed $\bar{v}_{r}(t)$ removed. All speeds normalized by $c$.

this scale here. The observed cross-shore flow is the forced, rather than freely propagating, response to tidal forcing of a rotating, stratified fluid over a sloping bottom. Though the frequency of these motions $\sigma$ is much higher than the frequency of free topographic Rossby or bottom-trapped waves, the forced response retains the bottom trapped character of these free modes.

\subsection{Baroclinic instability of the near-shore jet}

Figure 3 shows that the near-shore cyclonic jet transitions from a slowly accelerating feature with regular oscillations on the tidal time scale to a much more irregular flow that is no longer accelerating. The transition occurs at about $t \sigma / 2 \pi=90$ and the irregular nature of the jet persists throughout the remainder of the experiment.

Figure 7 is a series of snapshots from this second regime for $159<$ $t \sigma / 2 \pi<160$. Shown is the density field on a near-surface horizontal plane that slices through near-shore cyclonic jet and the corresponding time averaged, uplifted positions of the upper portion of the pycnocline shown in figure 4. Also shown are the positive and negative pressure contours. Over the fast time scale of a tidal period, the pressure contours rotate cyclonically making one full rotation while the density field changes relatively little. The azimuthal modal structure of the density field is approximately constant over 
a tidal cycle. The structure of the density field is consistent with baroclinic instability of the approximately geostrophically balanced cyclonic jet.

Orlanski and Cox (1972) give a scaling estimate for the azimuthal wavelength $\lambda_{\mathrm{bc}}$ of baroclinically unstable waves on a geostrophically balanced jet as

$$
\lambda_{\mathrm{bc}} \approx \frac{2}{L} \frac{N^{2} D^{2}}{f^{2}}
$$

where $L$ is the cross-shore scale over which the jet varies and $D$ is the total depth. Taking $N=N_{\text {pyc }}$ and $L=0.1 R$ gives an estimate for the azimuthal mode number $C_{0.95} / \lambda_{\mathrm{bc}}$ of approximately 12.3 where $C_{0.95}$ is the circumference at $r=0.95 R$. This estimate is slightly higher than the observed value of approximately 10 obtained by counting distinct dark spots in figure 7 (note the two ongoing mergers at about 10 and 4 o'clock in each figure), but is a reasonable match for a simple scaling argument.

While it is not obvious from the figure, the baroclinically unstable waves are evolving on a much slower time scale than the tidal period and adjacent cores, or dark spots in the density images, interact with one another and occasionally merge. During the period shown, this is occurring near $\theta=$ $3 \pi / 8$ and $-\pi / 8$. The slow evolution and modification of these features gives rise to the irregularity of the later portion of the time series in figure 3 .

\subsection{Near-shore eddies}

Baroclinic instability of the near-shore cyclonic jet produces slowly evolving vortical structures or near-shore eddies that provide a mechanism for cross-shore advection and transport. Figure 8 shows snapshots of the vertical vorticity $\zeta$ normalized by $f$, a measure of the local Rossby number characterizing the relative importance of the nonlinear terms to the Coriolis terms in the horizontal momentum equations. Throughout the interior of the domain, the magnitude of the vorticity is small, indicating that rotation dominates nonlinearity there and that the time scale of vortical motions is much longer than $1 / f$.

At the edges however, where distinct eddies are present, $|\zeta / f|$ exceeds 1 , indicating that nonlinearity is at least as important as rotation in the nearshore flow and that vortical and inertial time scales are comparable. The coherent vortices are cyclonic with high density cores and thus are examples of 'cold-core' eddies. They appear to slowly migrate cyclonically around the edge of the domain as they interact with one another. Blue bands with $\zeta / f<-1$ are regions where the total vertical vorticity $f+\zeta$ is anticyclonic.

Figure 9 shows a plan view of $w$ at $z / H=0.95$ at $t \sigma / 2 \pi=160$. The radial pattern in the basin interior is a signature of the forced waves at 


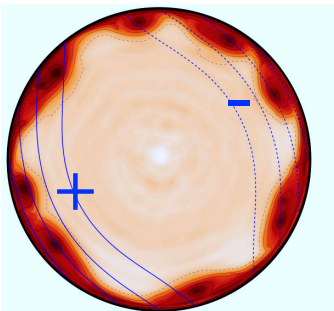

159.083

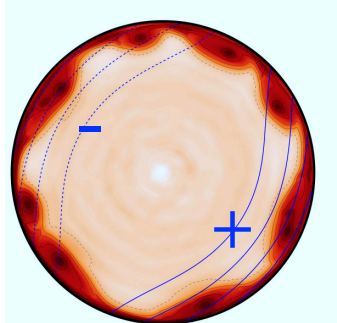

159.417

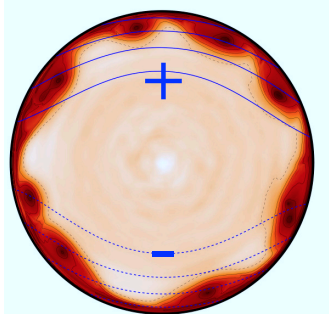

159.75

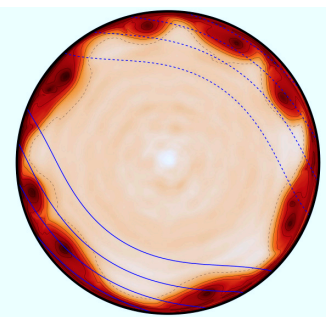

159.167

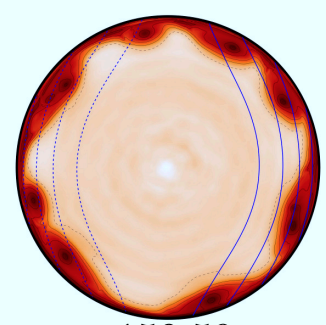

159.50

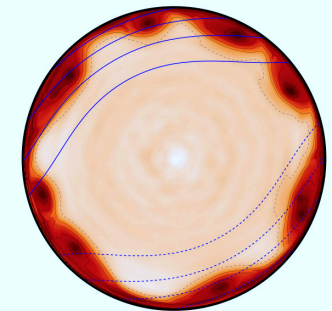

159.833

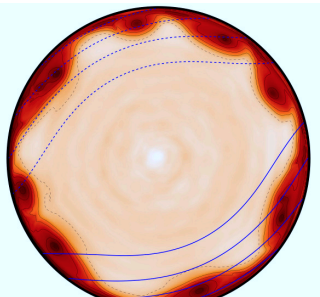

159.25

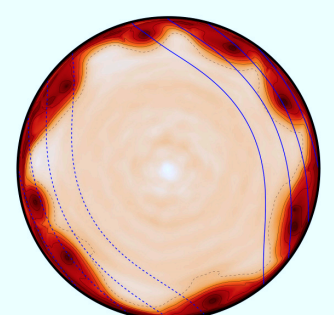

159.583

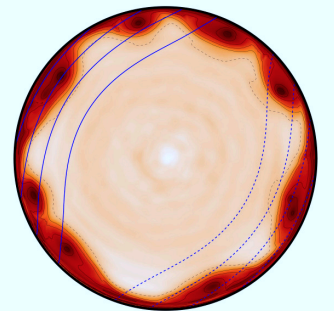

159.917

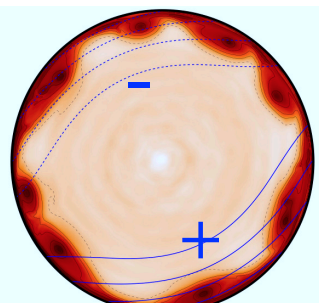

159.333

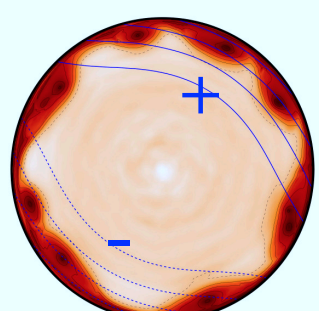

159.667

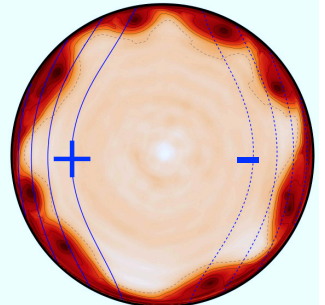

160.00

$$
\begin{array}{cccccc}
-0.05 & 0.00 & 0.05 & 0.10 & 0.15 & 0.20 \\
& & \rho^{\prime} / \Delta \rho & &
\end{array}
$$

Figure 7: Density at $z / H=0.95$ during last simulated tidal period. Also shown are positive (solid, +) and negative (dashed, -) pressure contours. Time, scaled by the tidal period, increases moving to the right and down. The phase of the pressure field propagates cyclonically while the eddies remain approximately stationary on the fast, tidal time scale. 
frequency $\sigma$ propagating inward from the edges. The radial wavelength corresponds roughly to $c / \sigma$. In addition to these signals, smaller scale waves are emitted from each of the near-shore vortices as indicated by the arrows. We have already noted that these motions stir the fluid at frequencies greater than $f$ and so it is not surprising radiating gravity waves would be excited, e.g. Danioux et al. (2012), Vanneste (2013) and references therein. As in Danioux et al. (2012), these waves are relatively weak: the frequency spectra shown in section 5.8 are dominated by motions at the forcing frequency and its harmonics.

\subsection{Along-shore structure near the coast}

The along-shore structure of the near-shore jet is shown in figure 10. The upper panel shows a snapshot of the azimuthal speed $v_{\theta}$ at $t \sigma / 2 \pi=160$, well after the jet has become baroclinically unstable. Distinct, nearly barotropic columns of relatively fast cyclonic flow correspond to the individual eddies apparent in figure 8. Between these eddies however, for example at $\theta=0$, fast azimuthal flow is confined to the lower half of the water column as was seen prior to the formation of the eddies and shown in figure 5 . The lower panel shows a snapshot of the azimuthal structure of the cross-shore flow $v_{r}$. The cross-shore flow has an azimuthal mode one structure and closely follows the temporal phase of the tidally forced pressure field which are shown in figure 8. The cross shore flow is on-shore, toward low near-shore pressure, around $\theta=0$ and off-shore, away from high near-shore pressure near $\theta= \pm \pi$. The presence of the eddies only slightly distorts this overall structure. The radial flow is bottom intensified with significant shear at about $z / H=0.9$. This near-bottom flow leads the overlying flow in phase by about $\pi / 4$ which appears to be largely responsible for the observed shear. The overall structure of $v_{r}$ shown here matches the cross-shore structure observed prior to the excitation of the eddies shown in figure 6 .

\subsection{Frequency spectra}

Time series of the azimuthal, radial and vertical components of velocity within the near-shore jet were extracted over time intervals preceding and following the instability of the jet and the formation of near-shore eddies. The dimensionless spectra $\mathcal{S}$ is shown as a function of dimensionless frequency $\omega / \sigma$ in figure 11 . The spectra are normalized such that

$$
\frac{1}{\sigma} \int \mathcal{S}(\omega) \mathrm{d} \omega=\frac{1}{T} \int \phi^{2}(t) \mathrm{d} t
$$



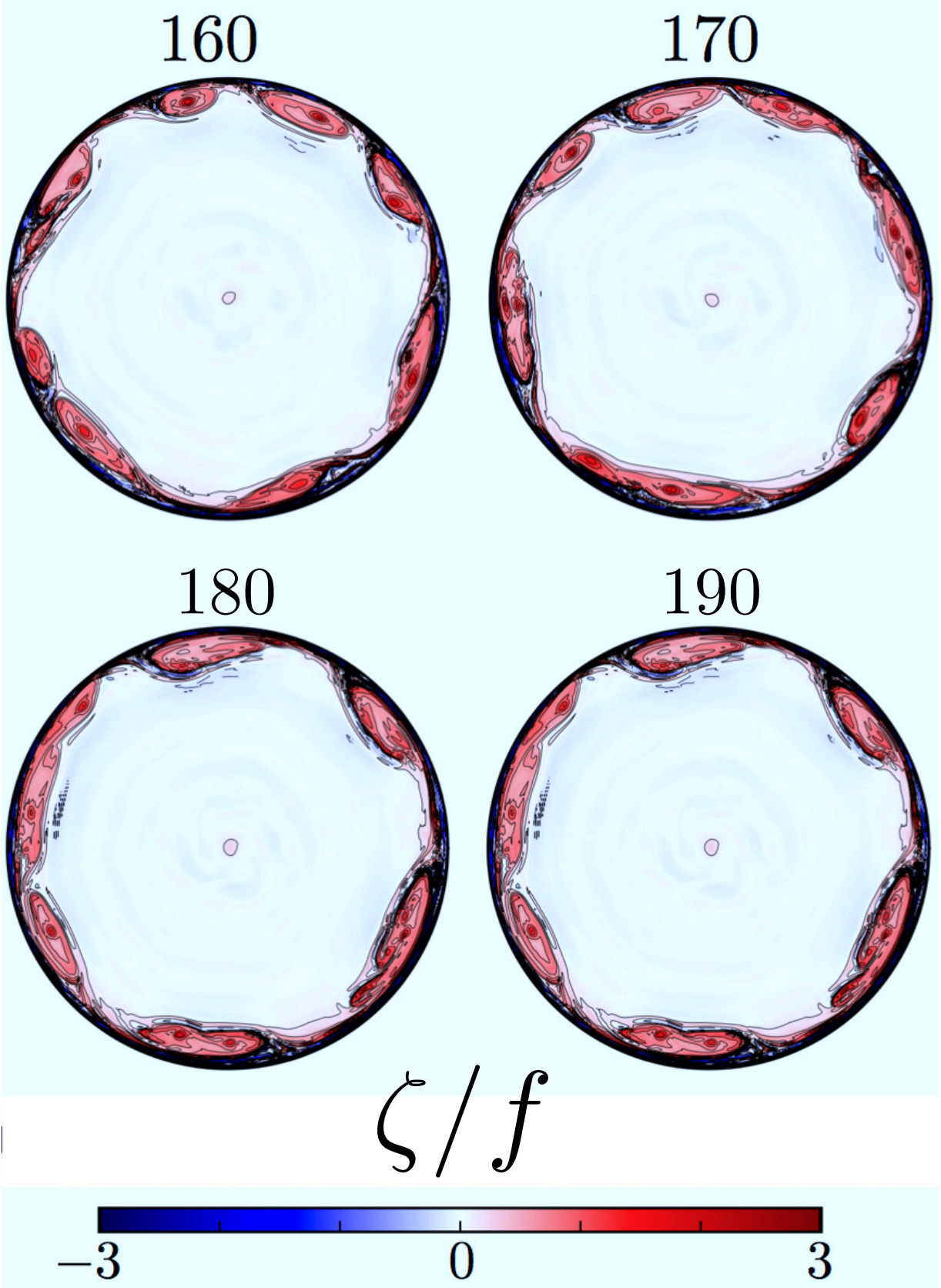

Figure 8: Vertical vorticity $\zeta=\frac{\partial v}{\partial x}-\frac{\partial u}{\partial y}$ normalized by $f$ at $z / H=0.95$ and at the $t \sigma / 2 \pi$ values of $160,170,180$ and 190 . 


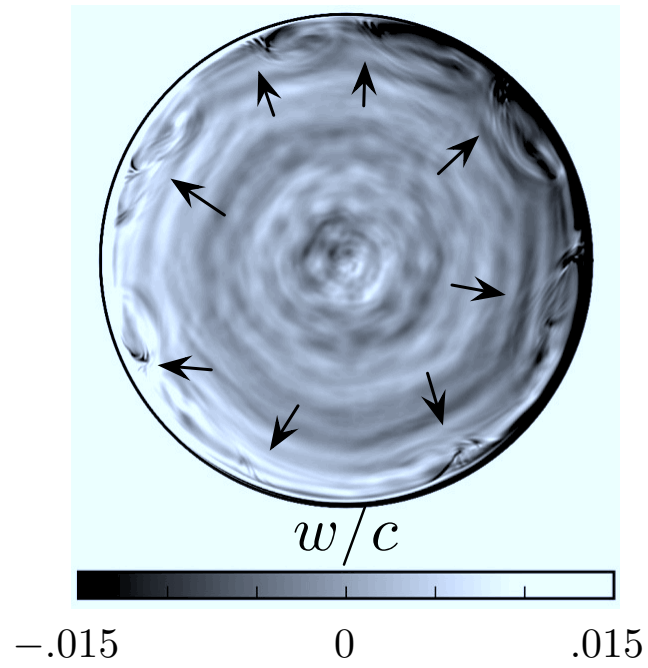

Figure 9: Vertical speed $w$ normalized by $c$ at $t \sigma / 2 \pi=160$ and $z / H=0.95$, corresponding to the last snapshot in figure 7 and the first in figure 8 . Arrows indicate regions of spontaneous emission of fast gravity waves.

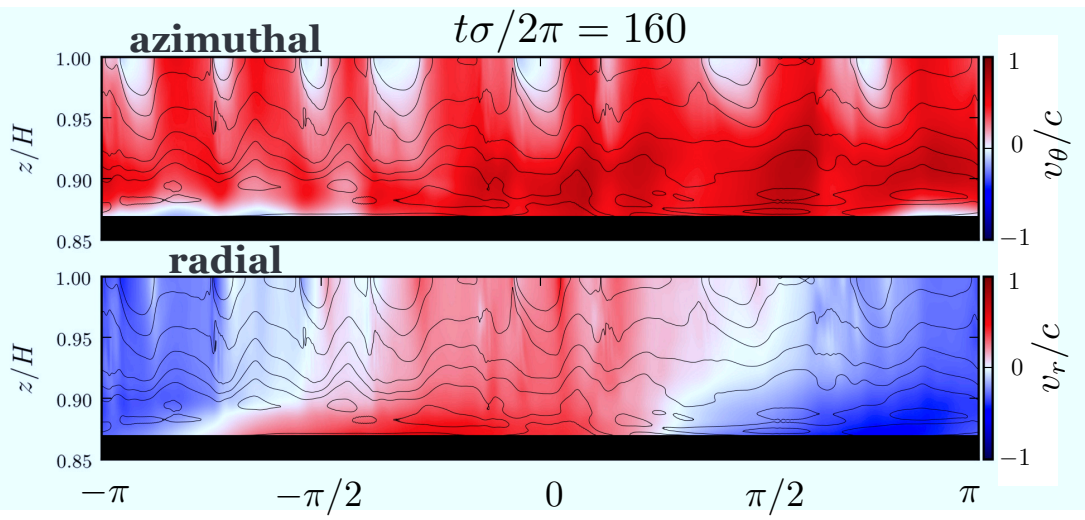

Figure 10: Top: Azimuthal speed $v_{\theta}(\theta, z / H) / c$ at $r / R=0.93$ at $t \sigma / 2 \pi=160$. Positive azimuthal speeds indicate cyclonic flow along the boundary. Bottom: Corresponding radial flow $v_{r} / c$. 

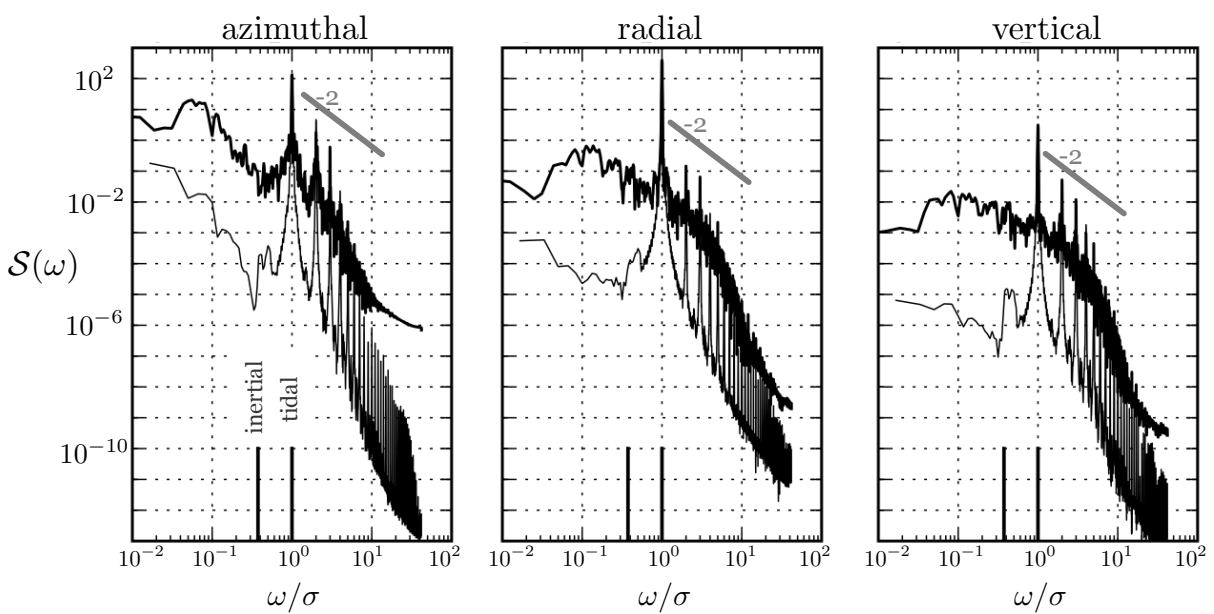

Figure 11: Frequency spectra for the azimuthal, radial and vertical currents at $\theta=\pi$, $x / R=-0.93$ and $z / H=0.925$. The spectra for the azimuthal speed corresponds to the lower time series shown in figure 3. Spectra are shown for two time intervals spanning 20-80 and 80-240 forcing periods. The inertial and tidal frequencies are indicated.

where $T$ is the time span of the interval and $\phi$ is the de-meaned, de-trended and windowed time series of speed values normalized by $c$.

Spectra corresponding to the time span prior to the excitation of eddies at about $\sigma t / 2 \pi=90$ are shown in thin lines. These spectra have distinct spectral lines at the tidal frequency $\omega / \sigma=1$ and at each tidal harmonic over a frequency range extending over more than a decade. Between the harmonics, there is very little variance. At the forced tidal frequency, the cross-shore component of the flow dominates and, across all frequencies, the horizontal flow speeds exceed vertical speeds.

Spectra computed after the onset of eddying motions are shown in dark lines. Compared to the earlier spectra, these spectra have significantly increased variance between the harmonic peaks and substantially more variance at low, sub-inertial frequencies. This is particularly true of the azimuthal spectra which has a broad maxima at frequencies about $f / 10$.

At frequencies higher than tidal but within the internal wave band $1<$ $\omega / \sigma<4.4$, the vertical and radial spectra for the later time period decay with frequency at a slope of approximately minus two. The corresponding azimuthal spectra falls off more rapidly with frequency.

Spectra of $w$ taken from near-shore and interior locations at the same 


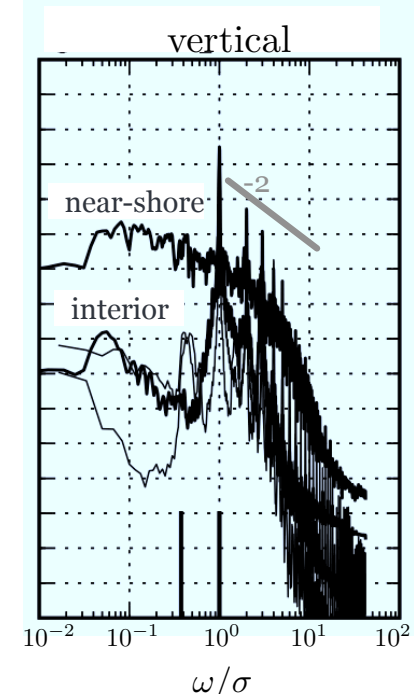

Figure 12: Comparison of near-shore $(x / R=-0.93)$ and interior $(x / R=-0.59)$ vertical velocity spectra at $\theta=\pi$ and $z / H=0.925$. Spectra are shown for the same time intervals as in figure 11.

height are shown in figure 12. Because the tidal forcing directly exerts only purely horizontal forcing, vertical motions in the interior pycnocline are a signature of radiation of internal waves from the near-shore boundaries. In comparison to the near-shore spectra, the interior spectra are reduced in strength by about two orders of magnitude in the internal wave band and about four orders of magnitude at sub-inertial frequencies.

\section{Discussion}

The interaction between nearly geostrophically 'balanced' motions, with slow time scales much longer than $1 / f$, and 'unbalanced' inertia-gravity waves with fast time scales of order $1 / f$ or smaller is a fundamental dynamical issue in both the atmosphere and the oceans. Often the interaction is cast in terms of the adjustment of a nearly balanced flow to or toward a balanced state via the radiation or spontaneous emission of fast inertia-gravity waves. Theories describing these interactions (quasi-geostrophic, semi-geostrophic etc) typically exploit a separation of time scales via the assumption of a small Rossby number $U / f L$, where $U$ and $L$ are the characteristic velocity 
and length scales of the nearly balanced motions and thus $L / U$ is the corresponding slow time scale. Often, but not always, these theories rely on the additional assumption of a separation in energies, with fast scale motions taken to be comparatively weak. Such low Rossby number flows have been shown to emit fast scale motions only weakly (Zeitlin, 2008).

Recent work e.g. (Zeitlin, 2008; Barkan et al., 2014) has examined the mechanisms by which rotating, stratified flows subject to steady large scale forcing can spontaneously develop localized features with sufficiently large Rossby numbers to bypass the theoretical constraints operative at small Rossby number and thereby generate fast, unbalanced motions more efficiently.

The problem discussed here is different in that the nearly balanced state is not the energy source for the fast, unbalanced wave motions. Rather, energy transferred from directly forced motions at the super-inertial tidal frequency drives the nearly balanced flow itself. The dynamics responsible for exciting and maintaining the slow, nearly balanced flow are fundamentally nonlinear and stem from the set-up of the pycnocline (figure 4) in the near-shore region of a shoaling basin. The slow flow takes the form of a cyclonic, near-shore, bottom-intensified jet (figure 5) that is prone to baroclinic instability (figure 7). The resulting vortices have Rossby numbers of order one (figure 8 ) and are therefore nonlinear motions with time scales $L / U$ of order $1 / f$. Interactions among these eddies transfer energy to larger scales and lower frequencies as the eddies merge.

The presence of these eddies alters the internal wave field by spontaneously emitting waves (figure 9) at frequencies comparable to the vorticity $\zeta$ and perhaps also by altering the background conditions within which nonlinear wave-wave interactions take place. The spectra in the gravity wave band, while spiky with peaks at the tidal frequency and its harmonics, are nevertheless continuous in frequency once the eddies have appeared (figures 11 and 12).

This problem is an example in which purely monochromatic forcing within the fast, internal wave frequency band produces a relatively rich flow with a nearly balanced along-shore jet, near-shore eddies and a broadbanded internal wave field. Compared to the trapped, non-propagating vortical motions near shore, the radiating inertia-gravity wave field in the interior is weak. Thus, in the absence of isolated topographic features, barotropic tidal forcing in a shoaling basin generates coastally amplified, energetic motions at tidal, sub-, and super-inertial frequencies, in addition to a much weaker internal wave field that radiates to the basin interior.

The bottom trapped cyclonic jet that develops in this simple experiment 
is in many ways similar to the subsurface, poleward flows observed along eastern ocean boundaries (Warren, 1990; Wooster and Reid, 1963). Such flows are ubiquitous at mid-latitudes in the major ocean basins (Smith, 1989), with perhaps the most comprehensive observations having been made off the west coast of North America (see e.g. Pierce et al 2000), where the undercurrent is located at approximately 200-275 $\mathrm{m}$ depth, 20-25 $\mathrm{km}$ offshore of the shelf break and has a mean speed of about $10 \mathrm{~cm} / \mathrm{s}$. While a convincing dynamical explanation for these poleward undercurrents has not yet emerged, most explanations invoke an along-shore pressure gradient of unknown origin, perhaps linked to regional wind gradients and/or local topographic features such as capes (Mooers, 1989). Given the seasonal and geographical persistence of these currents, and the expected complexity and variability of along-shore pressure gradients set up by wind and topography, such explanations should probably be taken tentatively.

As an alternative hypothesis, the dynamics illustrated here are intriguing, in part because they are generic. They rely neither on the wind nor on local topographic features yet provide a simple mechanism for driving cyclonic subsurface currents at continental slopes that is dynamically consistent with observations. While the tendency of the cyclonic jet modeled here to undergo baroclinic instability is reminiscent of the squirts, jets and eddies that are ubiquitous in eastern oceans, observed eddies originating in the California Undercurrent are about twice as likely to be anticyclonic than cyclonic (Collins et al., 2013), with the bias perhaps explained by topographic triggering near specific Points and Capes or nearshore bottom stress (Molemaker et al., 2015). Nevertheless, the idealized experiment discussed here shows that forced, tidal motions of a stratified fluid at sloping boundaries has the intrinsic tendency to 'set-up' a subsurface thermocline, drive or enhance a near-bottom cyclonic jet as a consequence of geostrophy, and ultimately, to produce along-shore variability and cross-shore transport via baroclinic instability. Such flows are the result of energy transfer from the surface tide to low-frequency currents and eddies.

Acknowledgements:

This work was supported by the National Science Foundation, grant number OCE-1155121. XSEDE computing resources were made available under grant TG-OCE120004. Discussions with Falk Feddersen, Clint Winant, Jim Lerczak, Jennifer Thomas and Roy Barkan are gratefully acknowledged and appreciated. 


\section{References}

Barkan, R., Winters, K. B., Smith, S. G. L., 2014. Energy cascades and loss of balance in a re-entrant channel forced by wind stress and buoyancy fluxes. J. Phys. Oceanogr. 45, 272-293.

Collins, C. A., Margolina, T., Rago, T. A., Ivanov, L., 2013. Looping RAFOS floats in the California Current System. Deep-Sea Res II 85, 42-61.

Csanady, G. T., 1967. Large-scale motion in the Great Lakes. J. Geophys. Res. 72 (16), 4151-4162.

Danioux, E., Vanneste, J., Klein, P., Sasaki, H., 2012. Spontaneous inertiagravity-wave generation by surface-intensified turbulence. J. Fluid Mech. 699, 153-173.

Garrett, C., 2003. Internal tides and ocean mixing. Science 301 (5641), 18581859.

Gerkema, T., Lam, F.-P. A., Maas, L. R., 2004. Internal tides in the Bay of Biscay: conversion rates and seasonal effects. Deep Sea Research Part II: Topical Studies in Oceanography 51 (25-26), 2995 - 3008.

Gill, A. E., 1982. Atmosphere-Ocean Dynamics. Academic Press, New York, $662 \mathrm{pp}$.

Hazewinkel, J., Winters, K. B., 2011. PSI of the internal tide on a $\beta$ plane: Flux divergence and near-inertial wave propagation. J. Phys. Oceanogr. 41, 1673-1682.

Huthnance, J. M., 1989. Internal tides and waves near the continental shelf edge. Geophysical \& Astrophysical Fluid Dynamics 48 (1-3), 81-106.

Li, Q., Farmer, D. M., 2011. The generation and evolution of nonlinear internal waves in the deep basin of the South China Sea. J. Phys. Oceanogr. $41,1345-1363$.

Longuet-Higgins, M. S., Stewart, R. W., 1964. Radiation stress in water waves; a physical discussion, with applications. Deep-Sea Res. 11, 529562 .

Mercier, M. J., Gostiaux, L., Helfrich, K., Sommeria, J., Viboud, S., Didelle, H., Ghaemsaidi, S. J., Dauxois, T., Peacock, T., 2013. Large-scale, realistic laboratory modeling of M2 internal tide generation at the Luzon Strait. Geophysical Research Letters 40 (21), 5704-5709. 
Merrifield, M. A., Holloway, P. E., 2002. Model estimates of M2 internal tide energetics at the Hawaiian Ridge. Journal of Geophysical Research: Oceans 107 (C8), 5-1-5-12.

Merrifield, M. A., Holloway, P. E., Johnston, T. M. S., 2001. The generation of internal tides at the Hawaiian Ridge. Geophysical Research Letters 28 (4), 559-562.

Molemaker, M. J., McWilliams, J. C., Dewar, W. K., 2015. Submesoscale instability and generation of mesoscale anticyclones near a separation of the California Undercurrent. J. Phys. Oceanogr. 45, 613-629.

Mooers, C. N., 1989. Workshop summary: Poleward flow-observational and theoretical issues. In: Poleward Flows Along Eastern Ocean Boundaries. Springer-Verlag, pp. 1-16.

Orlanski, I., Cox, M. D., 1972. Baroclinic instability in ocean currents. Geophysical Fluid Dynamics 4 (1), 297-332.

Ou, H. W., Bennett, J. R., 1979. A theory of the mean flow driven by long internal waves in a rotating basin, with application to Lale Kinneret. J. Phys. Oceanogr. 9, 1112-1125.

Pedlosky, J., 1987. Geophysical Fluid Dynamics. Springer-Verlag.

Pierce, S., Smith, R., Kosro, P. M., Barth, J. A., Wilson, C. D., 2000. Continuity of the poleward undercurrent along the eastern boundary of the mid-latitude north Pacific. Deep Sea Research Part II: Topical Studies in Oceanography 47 (5-6), 811-829-332.

Pingree, R., New, A., 1992. Abyssal penetration and bottom reflection of internal tidal energy in the Bay of Biscay. J. Phys. Oceanogr. 21, 28-39.

Poulin, F. J., Flierl, G. R., 2005. The influence of topography on the stability of jets. J. Phys. Oceanogr. 35, 811-825.

Poulin, F. J., Stegner, A., Hernandez-Arencibia, M., Marrero-Diaz, A., Sangra, P., 2014. Steep shelf stabilization of the bransfield coastal current: linear stability analysis. J. Phys. Oceanogr. 44, 714-732.

Rhines, P. B., 1970. Edge-, bottom-, and Rossby waves in a rotating, stratified fluid. Geophys. Fluid Dyn. 1, 273-302. 
Rudnick, D. L., Boyd, T. J., Brainard, R. E., Carter, G. S., Egbert, G. D., Gregg, M. C., Holloway, P. E., Klymak, J. M., Kunze, E., Lee, C. M., Levine, M. D., Luther, D. S., Martin, J. P., Merrifield, M. A., Moum, J. N., Nash, J. D., Pinkel, R., Rainville, L., Sanford, T. B., 2003. From tides to mixing along the Hawaiian Ridge. Science 301 (5631), 355-357.

Smith, R. L., 1989. Poleward flows along eastern ocean boundaries: An introduction and historical review. In: Poleward Flows Along Eastern Ocean Boundaries. Springer-Verlag, pp. 17-25.

Vanneste, J., 2013. Balance and spontaneous wave generation in geophysical flows. Annu. Rev. Fluid Mech. 45, 147-172.

Warren, B. A., 1990. Book Review: S.J. Neshyba, Ch.N.K. Mooers, R.L. Smith, R.T. Barber [eds.], Poleward flows along eastern ocean boundaries. Limnol. Oceanogr. 35(5), 1219-1220.

Winters, K. B., de la Fuente, A., 2012. Modelling rotating stratified flows at laboratory-scale using spectrally-based DNS. Ocean Modelling 49-50 (0), $47-59$.

Wooster, W. S., Reid, J. L., 1963. Eastern boundary currents. In: The sea. Vol. 2. Interscience, pp. 253-280.

Wunsch, C., 1971. Note on some Reynolds stress effects of internal waves on slopes. Deep Sea Research 18, 583-591.

Zeitlin, V., 2008. Decoupling of Balanced and Unbalanced Motions and Inertia-Gravity Wave Emission: Small versus Large Rossby Numbers. J. Atmos. Sci. 65, 3528-3542.

Zhao, Z., 2014. Internal tide radiation from the Luzon Strait. Journal of Geophysical Research: Oceans 119 (8), 5434-5448. 